\title{
Proteomics Study of the Effect of Left Atrial Appendage Resection on the Energy Metabolism of Atrial Muscle in Beagle Dogs with Rapid Atrial Pacing
}

\author{
Jing Chang \\ the Second Hospital of Jilin University \\ Yue Xing \\ the Second Hospital of Jilin University \\ Ting Yu \\ the Second Hospital of Jilin University \\ Xin Xue ( $\square$ xuexinsir@163.com ) \\ the Second Hospital of Jilin University
}

\section{Research Article}

Keywords: Atrial fibrillation, Left atrial appendage, Proteomics, Energy metabolism, Mitochondria

Posted Date: August 10th, 2021

DOI: https://doi.org/10.21203/rs.3.rs-753248/v1

License: (c) (i) This work is licensed under a Creative Commons Attribution 4.0 International License.

Read Full License 


\section{Abstract}

Aims The myocardial energy metabolism during atrial fibrillation $₫ A F \rrbracket$ is a research hotspot. Proteomics provides a new method for the study of atrial fibrillation; however, there are no related studies of the effect of the left atrial appendage $\triangle \mathrm{LAA} \rrbracket$ on the energy metabolism of the atrial muscle. We use proteomics to analyze the effect of resection of the LAA on the energy metabolism of the left atrial myocardial cells in beagle dogs with rapid atrial pacing.

METHODS Nine beagle dogs were divided into three groups: the model group (rapid atrial pacing/LAA resection), the positive control group (rapid atrial pacing/LAA preservation), and the negative control group (sinus rhythm). Twelve weeks later, the atrial tissues were resected for proteomics study. Parallel Reaction Monitoring (PRM) was used for the validation of the targeted proteomics.

RESULTS 55 proteins were upregulated and 68 proteins were downregulated in the experimental vs. the positive control group. Proteins related to glucose and lipid metabolism were mainly downregulated, and mitochondria-related proteins were mainly downregulated during rapid atrial pacing compared with sinus rhythm. After resection of the LAA, glucose-metabolism-related proteins showed a significant upregulation trend, lipid metabolism-related proteins were further downregulated, whereas mitochondriarelated proteins were upregulated compared with rapid atrial pacing with LAA preservation. PRM confirmed the reliability of the proteomics results.

CONCLUSION In the setting of AF, the resection of the LAA had a relatively large effect on the energy metabolism structure by affecting the quantity and functions of mitochondria.

\section{What's New}

1. In the context of the occurrence and development of $A F$, the resection of the LAA had a relatively large effect on the energy metabolism structure.

2. After the resection of the LAA, the overall glucose metabolism was significantly upregulated, while the overall lipid metabolism was further downregulated. Therefore, the LAA is likely to be the key in the regulation of the energy source of glucose under the condition of AF. The resection of the LAA may change the structure of the energy metabolism of $\mathrm{AF}$ by affecting the number and function of mitochondria.

3. The author have not found the literature on the effect of left atrial appendage on energy metabolism in myocardium.

\section{Introduction}

The heart is an organ with high energy consumption. Thus, an insufficient energy supply causes myocardial contraction and diastolic dysfunction, leading to the consumption of a large amount of 
energy because of the increase in electrical activity and contraction activity during AF, resulting in acute energy metabolism disorder. Moreover, structural changes in the energy metabolism may occur in the subsequent period, such as a decrease in the ratio of fatty acid metabolism and an increase in the ratio of glucose metabolism. The LAA, as an accessory structure of the left atrium, is considered to be a residual structure of the primitive atrium. With the improvement of the diagnosis and treatment of $A F$, greater attention has been paid to the LAA. As the LAA is accepted as the therapeutic target of AF in LAA occlusion, via resection and electrical isolation of this structure, would treatment of the LAA affect the left atrium, especially the energy metabolism of the atrial muscle?

Proteomics, as a popular study method, has been widely used by many scholars in atrial-fibrillationrelated research. For example, its application in the screening of predictors of AF revealed that the growth/differentiation factor 15 and resistin are markers that may contribute to the stratification of $A F$ risk in patients with cardiovascular disease. ${ }^{1}$ Barallobre-Barreiro et al. used proteomics to analyze glycoproteins in human cardiac extracellular matrix $(E C M)^{2}$ and found that the local bioavailability of anti-hypertrophic and pro-fibrotic growth factors can be adjusted. Previous proteomics studies ${ }^{3,4}$ performed using the atrial appendage tissues from patients as samples indicated that the atrial myocardial energy production is impaired in patients with valvular AF. It is suspected that the atrial appendage may be related to the energy metabolism of the atrial myocardium, and whether the effect of the atrial appendage on the energy metabolism of the atrial myocardium extends to the atrial muscle itself warrants further investigation.

Parallel reaction monitoring (PRM) is a targeted proteomics technology based on high-resolution and high-precision mass spectrometry that can selectively detect target proteins or peptides (such as peptides modified after translation), thus realizing absolute quantification of the target proteins/peptides.

In this study, proteomics methods were used to explore the effect of the resection of the LAA on the expression of proteins related to the energy metabolism in the left atrial tissue of beagle dogs with AF. Moreover, the PRM technology was used to verify the differentially expressed proteins quantitatively.

\section{Materials And Methods}

\section{Model preparation and grouping of beagle dogs with rapid atrial pacing}

For the purpose of establishing a sustained AF model, nine beagles purchased from Hunan Silaikejingda Experimental Animal Co.,Ltd囚China खwere fasted and deprived of water for $12 \mathrm{~h}$ before surgery. Routine disinfection of surgical devices and the surgical environment was used. After appeasement, dogs were anesthetized intraperitoneally with ketamine $(6-8 \mathrm{mg} / \mathrm{kg})$ combined with diazepam $(0.5-0.8 \mathrm{mg} / \mathrm{kg})$. The dogs were placed in the right lateral position and fixed on the experimental bench. Skin preparation, disinfection, and draping were performed on the chest and neck. Intubation using a 6F-sized endotracheal tube was used to connect the ventilator with proper adjusted parameters: tidal volume, $20 \mathrm{ml} / \mathrm{kg}$; 
respiratory rate, 20 times/min; and oxygen flow rate, $3.5 \mathrm{~L} / \mathrm{min}$. Subsequently, ketamine $(6-8 \mathrm{mg} / \mathrm{kg})+$ diazepam (0.5-0.8 $\mathrm{mg} / \mathrm{kg}$ ) were added intravenously every 15-40 min, according to the animal's reactions (respiration, eyelid membrane reflex, etc.); safe dosages of ketamine (50 mg/kg) and diazepam $(5 \mathrm{mg} / \mathrm{kg}$ ) were used. Blood pressure and ECG monitors were connected to limbs, and electrocardiographic monitoring was conducted during surgery.

A transverse incision with a length of about $5 \mathrm{~cm}$ was made in the 5th intercostal space of the left chest, to separate the skin, subcutaneous tissue, muscle, intercostal external muscle, intercostal internal muscle, pleura wall layer, and pleura visceral layer. The pericardium was opened and a pericardial hammock was made, to expose the LAA.

In the model group, three dogs had their LAAs removed; in the positive control group, the LAAs were preserved in three dogs; and in the negative control group, the pericardium of three dogs was directly sutured after opening, without removing the LAA or performing continuous pacing.

In the model group and the positive control group, the pacing electrode was sutured to the epicardium of the left auricular root with non-invasive suture, the ECG was monitored using a Powerlab system, and a canine pacemaker monitoring system was used to adjust the stimulation intensity of the pacemaker to twice the pacing threshold, using the pacing mode of $\mathrm{AOO}$ and a pacing frequency of $400 \mathrm{~min}^{-1}$. After adjustment to a satisfactory pacing waveform using the pacemaker, the epicardial pacing lead was fixed. The pericardium and thoracic cavity were checked for hemorrhage, and the chest wall was closed layer by layer. The subcutaneous tissue and skin were sutured after the chest cavity was aspirated, and a capsular bag was placed in the neck with implantation of an experimental high-frequency cardiac pacemaker. The tail end of the cardiac pacing electrode was connected with the buried high-frequency cardiac pacemaker through the subcutaneous tunnel. The neck of the dog was protected by a collar after surgery. After modeling, $3 \mathrm{~g} /$ day of cefazolin was administrated continuously for 5 days, to prevent infection. The dogs were supported by nutrition for 1 week, and their symptoms and signs were observed.

\section{Specimen acquisition}

After 12 weeks of continuous pacing, the experimental dogs were sacrificed via air injection in the jugular vein. The gross heart specimens of the dogs were taken and weighed and the left ventricle and left atrium were separated and weighed separately. The left atrial tissues (the free wall of left atrium, septum, pulmonary vein vestibule, and LAA) were collected in a sterile state and stored in liquid nitrogen, followed by transfer into a freezer set at $-80^{\circ} \mathrm{C}$, for future use.

\section{TMT quantitative proteomics}

\section{Protein extraction and digestion}


The frozen samples were ground to powder in liquid nitrogen. Four times the volume of lysis buffer (8 M urea, $1 \%$ protease inhibitor) was added, and ultrasonic lysis was performed on ice. The supernant was retained and the rediment was discarded after 10 min centrfugation. The protein concentration was measured using a BCA kit.

For digestion, the protein solution was treated with Dithiothreitol first for $30 \mathrm{~min}$ at $56^{\circ} \mathrm{C}$, followed by iodoacetamide for $15 \mathrm{~min}$ at room temprature. Then the urea in the sample was diluted to a concentration $<2 \mathrm{M}$ by $100 \mathrm{mM} \mathrm{NH}_{4} \mathrm{HCO}_{3}$. Finally, the first digestion was performed overnight at a mass ratio of 1:50 (trypsin: substrate), and the secondary digestion for $4 \mathrm{~h}$ at a mass ratio of 1:100 (trypsin: substrate).

\section{TMT labeling}

The trypsic peptides were desalted with Strata X C18 (Phenomenex), vacuum freeze dried, dissolved with 0.5 M TEAB and labeled according to the operating instructions of the TMT kit (Thermo Fisher Scientific). The labeled peptides were desalted after mixing and vacuum freeze dried.

\section{HPLC fractionation}

The peptids were fractionated by high pH reverse HPLC using the Agilent 300Extend C18 chromatographic column. The peptides were first separated into 60 components by a gradient of $8 \%$ to $32 \%$ acetonitrile $(\mathrm{pH} 9)$, and then combined into 14 components, followed by freeze-drying under vacuum.

\section{LC-MS/MS analysis}

The peptides were dissolved in mobile phase A ( $0.1 \%$ formic acid and $2 \%$ acetonitrile) and separated using an EASY-nLC 1000 ultra-high-performance liquid system. 0.1\% formic acid and $90 \%$ acetonitrile. The gradient was set as follows: $0-26 \mathrm{~min}, 9 \%-23 \%$ mobile phase B $(0.1 \%$ formic acid and $90 \%$ acetonitrile); $26-34 \mathrm{~min}, 23 \%-35 \%$ mobile phase $\mathrm{B} ; 34-37 \mathrm{~min}, 35 \%-80 \%$ mobile phase $\mathrm{B}$; and $37-40$ min, $80 \%$ mobile phase $B$, with the flow rate maintained at $400 \mathrm{~nL} / \mathrm{min}$.

Then the peptides were injected into NSI ion source and analyzed by Q Exactive Orbitrap Fusion mass spectrometry. The scanning range of the primary mass spectrometry was set to $400-1500 \mathrm{~m} / \mathrm{z}$, with the scanning resolution set to 60,000 , while the scanning range of the secondary mass spectrometry was fixed at $100 \mathrm{~m} / \mathrm{z}$, with the secondary scanning resolution set to 30,000. A data-dependent procedure that alternated between one MS scan followed by $20 \mathrm{MS} / \mathrm{MS}$ scans with 30.0 s dynamic exclusion. The

\section{Database Search}


Maxquant (v1.5.2.8) was used to search against dog uniprot database (CANIS_LUPUS_FAMILIES_9615_PR_20191025, 25496 sequences) concatenated , with reverse decoy database. Trypsin/P was set as cleavage enzyme allowing up to 2 missing cleavages. The mass tolerance for precursor ions was set as $20 \mathrm{ppm}$ in First search and $5 \mathrm{ppm}$ in Main search, and the mass tolerance for fragment ions was set as $0.02 \mathrm{Da}$. The cysteine alkylation was set as a fixed modification, and the oxidation of methionine, $\mathrm{N}$-terminal acetylation of proteins, and deamidation (NQ) set as variable modifications. The quantitative method was set as TMT-10plex, with the FDR for protein identification and PSM identification set to $1 \%$.

Significant changes in protein expression were indicated using fold changes of $>1.2$ or $<0.8$ as cut-off values. A double-tailed Fisher's exact test was adopted, and the standard error detection rate control method was used to correct the multiple-hypothesis test. After the correction, significance was set at $P<$ 0.05 .

\section{Bioinformatic Analysis}

WoLF PSORT (https://wolfpsort.hgc.jp/) was used to annotate the submitted proteins regarding subcellular localization. The functional annotation of each protein was performed against COG/KOG database (http://www.ncbi.nlm.nih.gov/COG), UniProt-GOA database (http://www.ebi.ac.uk/GOA/), and KEGG pathway database. For pathway enrichment analysis, a two-tailed Fisher's exact test was employed for the enrichment analysis of the differentially expressed proteins against all identified proteins. The terms with $p$ values below 0.05 were treated as significant.

\section{Validation of differentially expressed proteins}

Parallel reaction monitoring (PRM) analyses were performed using a Q Exactive ${ }^{\mathrm{TM}}$ Plus (Thermo) coupled online to the UPLC. The resulting MS data were processed using Skyline (v.3.6). Peptides parameters: enzyme was set to Trypsin [KR/P], max missed cleavage set as 0 , the peptide length set as $7-25$, and the cysteine alkylation set as a fixed modification. Transition parameters: the parent ion charges were set as 2,3 , the daughter ion charge set as 1 , and the ion types set to $b, y$. The product ion selection started from the third to the last, with the ion match tolerance set to $0.02 \mathrm{Da}$.

\section{Results}

\section{Differentially expressed proteins in the experimental group compared with the positive control group and the negative control group}


In this study, 4048.0 proteins were identified, of which 3814.0 proteins contained quantitative information. The adoption of a threshold of change in differential expression of 1.2 -fold and a $P$-value $<$ 0.05 in a $t$-test as the significance threshold revealed that, among the quantified proteins, 55 proteins were upregulated and 68 proteins were downregulated in the experimental group vs. the positive control group. In the analysis of the experimental group vs. the negative control group, 166 proteins were upregulated and 87 proteins were downregulated. Finally, 577 proteins were upregulated and 287 proteins were downregulated in the positive control group vs. the negative control group (Figure 1).

\section{Subcellular localization classification}

In the experimental group vs. the positive control group analysis, $29.27 \%$ (36) of the differentially expressed proteins were located in the cytoplasm, $23.58 \%$ (29) were located in mitochondria, $19.51 \%$ (24) were located in the nucleus, and the remaining proteins were located in the plasma membrane, endoplasmic reticulum, and extracellular matrix (Figure 2A).

\section{COG/KOG functional classification}

In the experimental group vs. the positive control group analysis, according to the KOG categories, related proteins with clear functions and more than five differentially expressed proteins included the following: 13 proteins were related to energy production and conversion; five proteins were related to carbohydrate transport and metabolism; 12 proteins were related to lipid transport and metabolism; seven proteins were related to translation, ribosome structure, and biogenesis; 10 proteins were related to posttranslational modification, protein turnover, and chaperones; 13 proteins were related to signal transmission mechanisms; and eight proteins were related to the cycloskeleton (Figure 2B).

\section{GO enrichment analysis}

Enrichment associated with glucose metabolism in the biological process of differential protein upregulation was significant in the experimental group compared with the positive control group, as was the enrichment of mitochondrial ATP metabolism, including the glucose catabolic process, the glycolytic process through glucose-6- phosphate, the glycolytic process through fructose-6-phosphate,

mitochondrial translational elongation, translational termination, mitochondrial respiratory chain complex assembly, and the ATP metabolic process (Figure 3A). In contrast, enrichment of lipid metabolism was significant in the bioprocess of differential protein downregulation, including the regulation of the phospholipid biosynthetic process, the fatty-acyl-CoA biosynthetic process, the long-chain fatty-acyl-CoA biosynthetic process, the long-chain fatty-acyl-CoA metabolic process, the regulation of the cholesterol metabolic process, sterol homeostasis, and cholesterol homeostasis (Figure 3B). 
In the experimental group vs. positive control group analysis, mitochondrial and respiratory chain cell components were enriched among the differentially upregulated proteins (Figure 4A), whereas lipid particle enrichment was significant in the bioprocess of downregulation (Figure 4B).

\section{KEGG pathways analysis}

In the experimental group vs. positive control group analysis, the significant protein enrichment associated with KEGG pathways of differential protein upregulation included the glucagon signaling pathway, Oxidative phosphorylation, Glycolysis Gluconeogenesis (Figure 5A). In contrast, the significant enrichment of downregulated KEGG pathways included the Biosynthesis of unsaturated fatty acids, 2Oxocarboxylic acid metabolism, PPAR signaling pathway, Regulation of lipolysis in adipocytes, Citrate cycle, and the AMPK signaling pathway (Figure 5B)

The comparison of the positive and the negative control groups did not show the significant upregulation of protein-enriched pathways closely related to energy metabolism with respect to biological process, cell components, and KEGG pathway. However, the area corresponding to the differentially downregulated protein enrichment was obvious and included NADH dehydrogenase complex assembly, mitochondrial respiratory chain complex assembly in biological process, mitochondrial translational elongation, mitochondrial translation, fatty acid beta-oxidation, and the ATP metabolic process (Figure 6A). In turn, we found a significant enrichment of differentially downregulated proteins associated with mitochondria in cell components (Figure 6B); the enrichment of differentially downregulated proteins associated with Oxidative phosphorylation, fatty acid degradation, glucagon signaling pathway, and glycolysis/gluconeogenesis in KEGG pathways was also significant (Figure 6C).

\section{PRM verification}

Twenty differentially expressed proteins related to glycolipid metabolism from nine samples were selected for PRM quantitative verification: however, only 15 of them were quantified because of the limitations of the protein features and abundance (Table 1). The PRM results were in line with the variation trend of previous proteomics studies, which confirmed the reliability of the proteomics results.

\section{Discussion}

The heart is a high energy-consuming organ, and AF, as a rapid type of arrhythmia, substantially increases electrical activity and mechanical systole; therefore, in this context, the heart needs to consume large amounts of ATP. ${ }^{5}$ The main source of ATP is the catabolic metabolism of sugars, lipids, lactic acid, ketone bodies, amino acids, etc. Under normal conditions, the main source of energy for myocardial activity is the oxidative catabolism of fatty acids, especially $\beta$-oxidation. 
In addition, previous studies have shown that the expression of atrial muscle glycolysis and pyruvate metabolism-associated proteins is decreased in patients with $\mathrm{AF}^{3,6,7}$ while the abundance of lipidmetabolism-associated proteins is decreased. ${ }^{6}$ Our proteomics study also indicated that proteins associated with glycolysis and fatty acid degradation are downregulated in AF compared with sinus rhythm, which is in agreement with a previous study; however, compared with AF alone, fatty acid metabolism is further downregulated after the resection of the LAA under the condition of AF, while proteins in the glucose metabolism process, such as glycolysis or oxidative phosphorylation, were upregulated. This suggests that the LAA is key in the regulation of carbohydrate energy sources under the condition of AF.

Based on the results of our study, we can speculate that the structure of the atrium muscle cell energy metabolism has changes dramatically after the resection of the LAA in the presence of AF, with a further decline in lipid metabolism and upregulation of glucose metabolism. According to the literature, the mechanism of energy metabolism disorder is relevant in AF, with decreased adenine nucleotides, decreased high energy phosphate, reduced activity of enzymes that are pertinent to energy metabolism, as well as changed mitochondrial oxidative regulation. ${ }^{3,8}$ How does the resection of the LAA affect the energy metabolism of the atrial muscle under the condition of AF? Further analysis of the proteomics results revealed a significant trend toward the upregulation of protein expression in association with mitochondria after the resection of the LAA; moreover, mitochondrial translational elongation, translational termination, mitochondrial respiratory chain complex assembly, and several other biological processes related to upregulated proteins were also apparent. In turn, the analysis of cell components revealed a relationship between the respiratory chain complex, mitochondrial ribosome, respiratory chain, mitochondrial respiratory chain, inner mitochondrial membrane protein complex, mitochondrial protein complex, mitochondrial inner membrane, mitochondrial membrane, mitochondrial envelope, mitochondrial part, mitochondrial matrix, and mitochondrion and significantly upregulated proteins. This finding suggests the crucial function of mitochondria in the LAA in the process of the energy metabolism of AF. It is speculated that the resection of the LAA should improve the amount and function of mitochondria in atrial muscle cells. Montaigne et al. found that mitochondrial function disorder is closely related to the onset and maintenance of $A F^{9}{ }^{9}$ The sarcK-ATP channel is highly sensitive to mitochondrial energy metabolism, and mitochondrial dysfunction causes an increase in the opening of the sarcK-ATP channel, which affects regional myocardial conductivity and facilitates the occurrence of AF. In addition, mitochondrial dysfunction leads to the deficiency of ATP synthesis, affects the $\mathrm{Na}^{+}-\mathrm{K}^{+}$-ATP pump, causes $\mathrm{Ca}^{2+}$ overload in myocardial cells, and facilitates the occurrence and development of AF. Conversely, mitochondrial reactive oxygen species (ROS) may damage atrial muscle cells, ${ }^{10}$ affect enzyme activity, hinder electrical conductivity, and restrain ATP synthesis, and can induce ROS release and mitochondrial overload with positive feedback. AF may also be caused by the damage of $\mathrm{Ca}^{2+}$ balance.

The analysis of our proteomics results showed that both glycolysis and glucose oxidation were significantly increased after the resection of the LAA. The provision of ATP by glucose oxidation should be conducted in mitochondria, and the mitochondria-related protein expression is upregulated, while lipid 
metabolism expression from another energy source is downregulated, which confirms the upregulation of glucose aerobic metabolism as a side process. It also showed that proteins related to a bioprocess (NADH dehydrogenase complex assembly) were upregulated after the resection of the LAA. In this process, NADH is a product of glucose oxidation, indicating that glucose aerobic metabolism is also upregulated. In the glycolytic pathway, the glycolytic process through glucose-6-phophate and the glycolytic process through fructose-6-phosphate were increased significantly, showing that the increase in the glucose oxidation metabolism alone represents a failure to meet the myocardial energy requirement under the condition of AF after the resection of the LAA, and that glycolysis is needed as a supplement. However, excessive glycolysis can lead to the accumulation of pyruvic acid, which cannot be entirely oxidized; thus, it accumulates and is transformed into lactic acid, causing cell acidosis and ischemia.

In the case of fasting, about $70 \%$ of the cardiac energy is derived from fatty acid oxidation; thus, lipid metabolism plays an important part in the energy metabolism of myocardial cells. ${ }^{11}$ The present study suggests that the fatty acid metabolism is downregulated in the context of AF and, to an even greater extent, after the resection of the LAA. It is known that ATP is mainly provided by fatty acid oxidation through the tricarboxylic acid cycle, of which the mitochondria is the primary site. Mitochondriaassociated proteins and pathways were upregulated after the resection of the LAA; thus, why did downregulation of lipid metabolism occur, and was even more prominent than that observed in simple AF? A careful analysis of our proteomics results revealed that the AMPK and PPAR signaling pathways were downregulated, which may be the cause of this phenomenon. The downstream lipid metabolism pathway can be regulated after the activation of AMPK phosphorylation, thus increasing the expression of FAT/CD36 on the myocardial cell membrane and fatty acid uptake. ${ }^{6}$ However, the AMPK signaling pathway was downregulated, indicating a decrease in fatty acid uptake. Although the expression of mitochondria-related proteins was increased, lipid materials were reduced, with the combined end result being the downregulation of lipid metabolism. The increase in PPAR a expression may lead to a significant increase in fatty acid uptake, $\beta$ oxidation, and lipid load in cardiomyocytes; conversely, lipid metabolism may be decreased. Mice lacking PPAR a show a decrease in the fatty acid oxidation rate and an increase in the glucose oxidation rate of the heart. ${ }^{12}$ The decrease in lipid metabolism may also lead to lipid deposition in cells. Studies have shown that CPT-1 is the rate-limiting enzyme in the metabolism of fatty acids and can catalyze the conversion of long-chain acyl coenzyme A to long-chain acyl carnitine, which is then transported to mitochondria. CPT-1 was downregulated in the atrium of a rabbit model of $\mathrm{AF}$ and may be involved in the formation of lipid droplets in cardiomyocytes caused by fat metabolism. ${ }^{13}$ Similar results were obtained in our proteomics study. In AF compared with sinus rhythm, proteins related to the oxidation process of atrial cell fatty acid $\beta$ were downregulated, and proteins related to the fatty acid degradation KEGG pathway were also downregulated, suggesting a decrease in fatty acid consumption. As shown in Fig. 5A, lipid particles were downregulated significantly after the resection of the LAA for AF, indicating that the resection of the LAA can reduce lipid deposition, which may be related to the downregulation of lipid anabolism rather than that of lipid catabolism. Because lipid metabolism requires more oxygen to produce the same amount of ATP than does the sugar oxidation pathway, the downregulation of lipid metabolism is beneficial regarding myocardial oxygen consumption during AF. 
Among the proteins that were verified by PRM, we found many key proteins involved in glycolipid metabolism, such as H9GW55 and F1PDJ0. The results of PRM indicate that the TMT results and PRM results for these two specific proteins exhibited exactly the same change trend in different groups, indicating that the differentially expressed proteins identified in the proteomics study are credible. A search of the https://www.uniprot.org/ website shows that H9GW55 is a phosphoglycerate mutase and a key enzyme in the sugar metabolism process, as it catalyzes the mutual conversion between 3phosphoglyceric acid and 2-phosphoglyceric acid and is involved in the glycolytic process. The expression of H9GW55 in AF was significantly lower than that observed in sinus rhythm. However, after the resection of the LAA, the expression of this protein was significantly higher than that detected in animals with AF and preservation of the LAA. Even after the resection of the LAA, there was no obvious difference in the expression of this protein compared with the sinus rhythm condition. Resection of the LAA leads to the positive regulation of the expression level of the protein in the left atrial muscle cells and the improvement of glucose metabolism. F1PDJ0, however, is an apolipoprotein C-III protein that is involved in many aspects of lipid metabolism. F1PDJ0 can negatively regulate cholesterol for direct entry into cells or organelles, prevent or reduce the formation of fatty acids, stop or reduce the activity of lipoprotein lipase, negatively regulate the decomposition of triglycerides, etc. Our PRM verification results showed that this protein was significantly improved under the condition of AF compared with sinus rhythm. In turn, after the resection of the LAA, the level of expression of this protein in the left atrial myocardium will continue to increase compared with that observed during AF; thus, F1PDJ0 has a negative regulatory effect on lipid metabolism. Therefore, the upregulation of this protein indicates that resection of the LAA will further downregulate the lipid metabolism level.

It can be concluded from this study that the simple resection of the LAA can produce a significant impact on the energy metabolism structure of the atrial muscle, suggesting that the LAA is a key regulatory site of the energy metabolism of the atrial muscle. It is currently uncertain whether the left atrial hemodynamics will be affected by this change in the energy metabolism structure or cause structural remodeling. Additional studies are necessary to clarify these issues.

\section{Conclusion}

This study showed that, in the context of the occurrence and development of $A F$, the resection of the LAA had a relatively large effect on the energy metabolism structure. After the resection of the LAA, the overall glucose metabolism was significantly upregulated, while the overall lipid metabolism was further downregulated. Therefore, the LAA is likely to be key in the regulation of the energy source of glucose under the condition of AF. The resection of the LAA may change the structure of the energy metabolism of $\mathrm{AF}$ by affecting the number and function of mitochondria.

\section{Abbreviations}

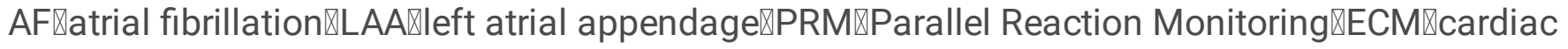

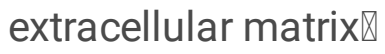




\section{Declarations}

All experimental protocols were approved by Hunan Drug Safety Evaluation Research Center. All methods were carried out in accordance with relevant guidelines and regulations and reported in accordance with ARRIVE guidelines .

All authors gave consent to publication.

All data generated or analysed during this study are included in this published article.

There were non-financial competing interests in this study.

This work is supported in part by funds provided by Technology Research Project of Jilin Province Department of Education (grant number JJKH20190054KJ).

\section{Authors' contributions:}

Chang Jing: :Proteomic experiments

\section{Conflict of interest statement}

The authors declared that they have no conflicts of interest to this work. We declare that we do not have any commercial or associative interest that represents a conflict of interest in connection with the work submitted.

Xing Yue : Writing articles

Yu Ting $\bowtie$ Bioinformatics analysis and statistical analysis

Xue Xin $\bowtie$ Pacemaker surgery and ultrasound examination

Thanks for Dr. Guirong Zeng from Hunan Drug Safety Evaluation Research Center. He gave us a great help with the animal's experiment.

\section{References}

1. Doulamis IP, Samanidis G, Tzani A, Antoranz A, Gkogkos A, Konstantopoulos P, et al. Proteomic profile of patients with atrial fibrillation undergoing cardiac surgery. Interact Cardiovasc Thorac Surg2019; 28: 94-101. 
2. Barallobre-Barreiro J, Gupta SK, Zoccarato A, Kitazume-Taneike R, Fava M, Yin X, et al. Glycoproteomics Reveals Decorin Peptides With Anti-Myostatin Activity in Human Atrial Fibrillation. Circulation2016; 134: 817-832.

3. Tu T, Zhou S, Liu Z, Li X, Liu Q. Quantitative proteomics of changes in energy metabolism-related proteins in atrial tissue from valvular disease patients with permanent atrial fibrillation. Circ J2014; 78: 993-1001.

4. Bai F, Tu T, Qin F, Ma Y, Liu N, Liu Y, et al. Quantitative proteomics of changes in succinylated proteins expression profiling in left appendages tissue from valvular heart disease patients with atrial fibrillation. Clin Chim Acta2019; 495: 345-354.

5. Ashrafian H, Neubauer S. Metabolomic profiling of cardiac substrate utilization: fanning the flames of systems biology? Circulation2009; 119: 1700-1702.

6. Lenski M, Schleider G, Kohlhaas M, Adrian L, Adam O, Tian Q, et al. Arrhythmia causes lipid accumulation and reduced glucose uptake. Basic Res Cardio/2015; 110: 40.

7. Thijssen VL, Ausma J, Borgers M. Structural remodelling during chronic atrial fibrillation: act of programmed cell survival. Cardiovasc Res2001; 52: 14-24.

8. Sun DM, Yuan X, Wei H, Zhu SJ, Zhang P, Zhang SJ, et al. Impaired myocardium energetics associated with the risk for new-onset atrial fibrillation after isolated coronary artery bypass graft surgery. Coron Artery Dis2014; 25: 224-229.

9. Montaigne D, Maréchal X, Lacroix D, Staels B. From cardiac mitochondrial dysfunction to clinical arrhythmias. Int J Cardio/2015; 184: 597-599.

10. Mayr M, Yusuf S, Weir G, Chung YL, Mayr U, Yin X, et al. Combined metabolomic and proteomic analysis of human atrial fibrillation. J Am Coll Cardiol2008; 51: 585-594.

11. Lopaschuk GD, Ussher JR, Folmes CD, Jaswal JS, Stanley WC. Myocardial fatty acid metabolism in health and disease. Physiol Rev2010; 90: 207-258.

12. Madrazo JA, Kelly DP. The PPAR trio: regulators of myocardial energy metabolism in health and disease. J Mol Cell Cardiol2008; 44: 968-975.

13. Liu Y, Geng J, Li Y, Shen J, Xiao X, Sheng L, et al. ß3-adrenoceptor mediates metabolic protein remodeling in a rabbit model of tachypacing-induced atrial fibrillation. Cell Physiol Biochem2013; 32 : 1631-1642.

\section{Table}

Due to technical limitations, table 1 is only available as a download in the Supplemental Files section.

\section{Figures}




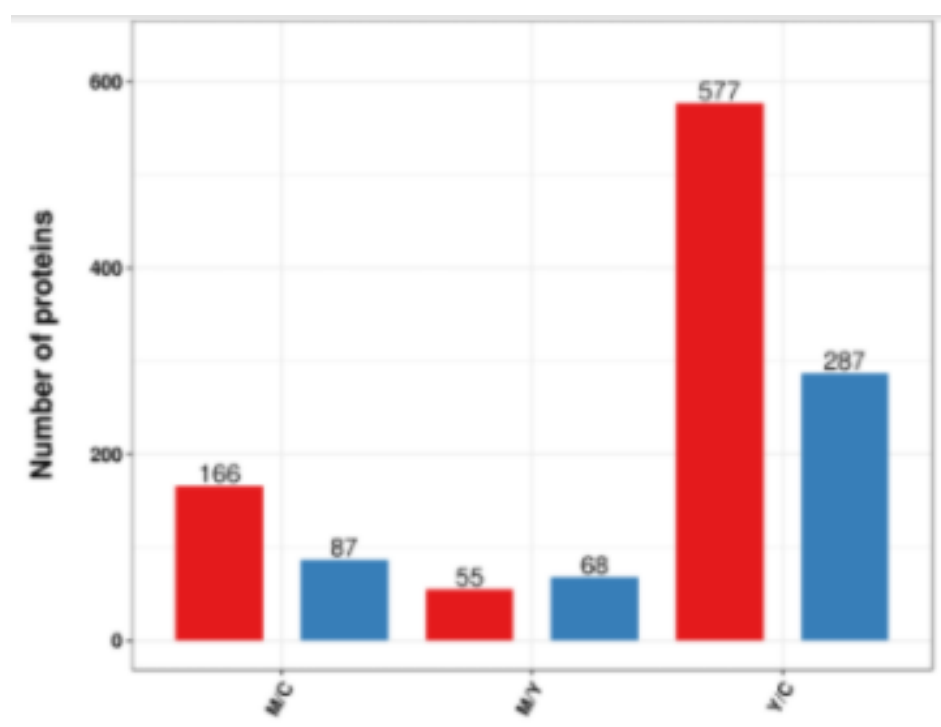

Comparable groups
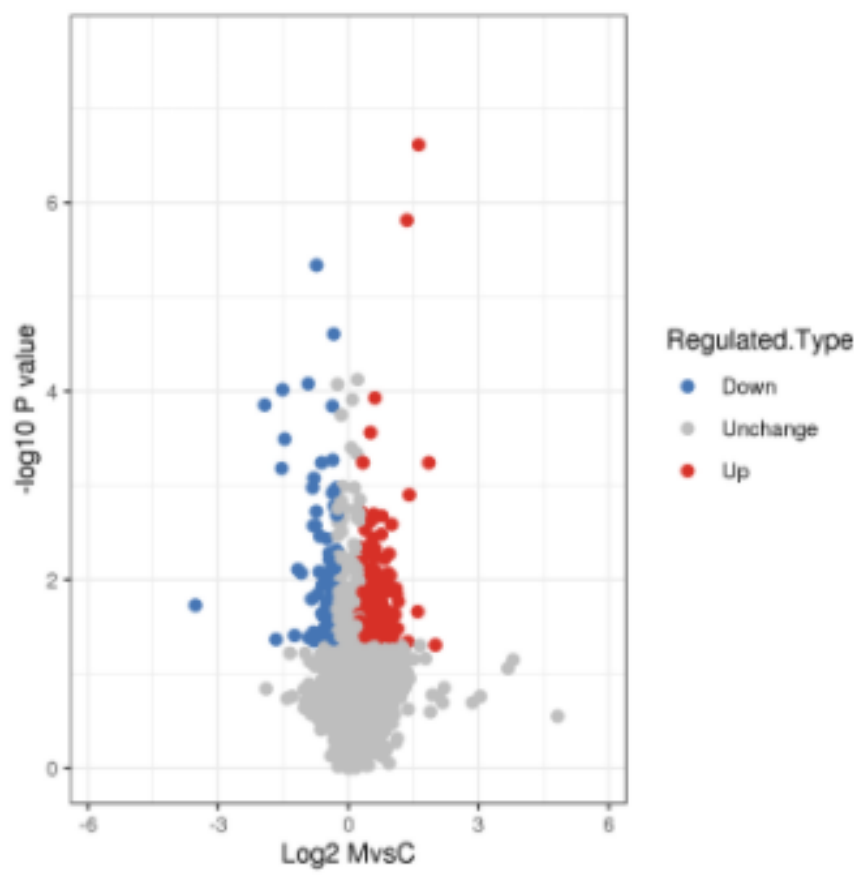

C

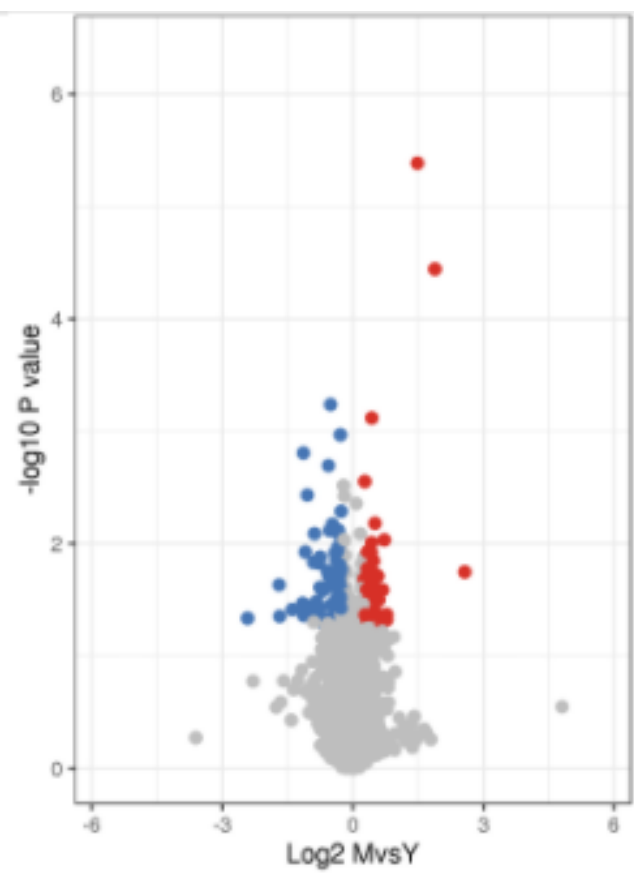

B

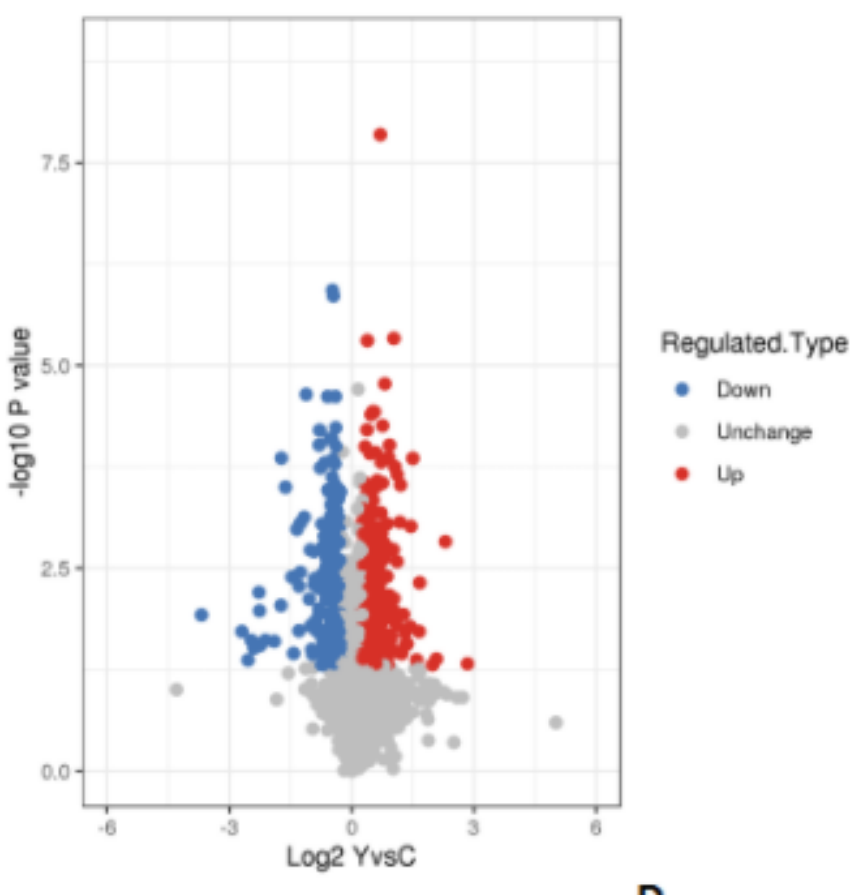

D

\section{Figure 1}

M: experimental group; Y: positive control group; C: negative control group. A: Number of differentially expressed proteins in each group. B: Quantitative volcano plot of differentially expressed proteins in the experimental group vs. the positive control group. C: Quantitative volcano plot of differentially expressed proteins in the experimental group vs. the negative control group. D: Quantitative volcano plot of differentially expressed proteins in the positive control group vs. the negative control group. 


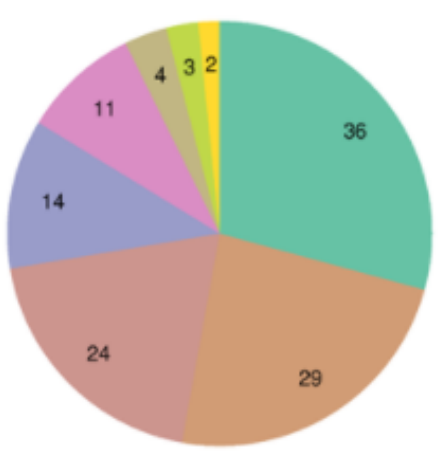

cytoplasm (29.27\%)

mitochondria $(23.58 \%)$

nucleus (19.51\%)

extracellular $(11.38 \%)$

plasma membrane $(8.94 \%)$

cytoplasm,nucleus $(3.25 \%)$

endoplasmic reticulum (2.44\%)

other $(1.63 \%)$

A

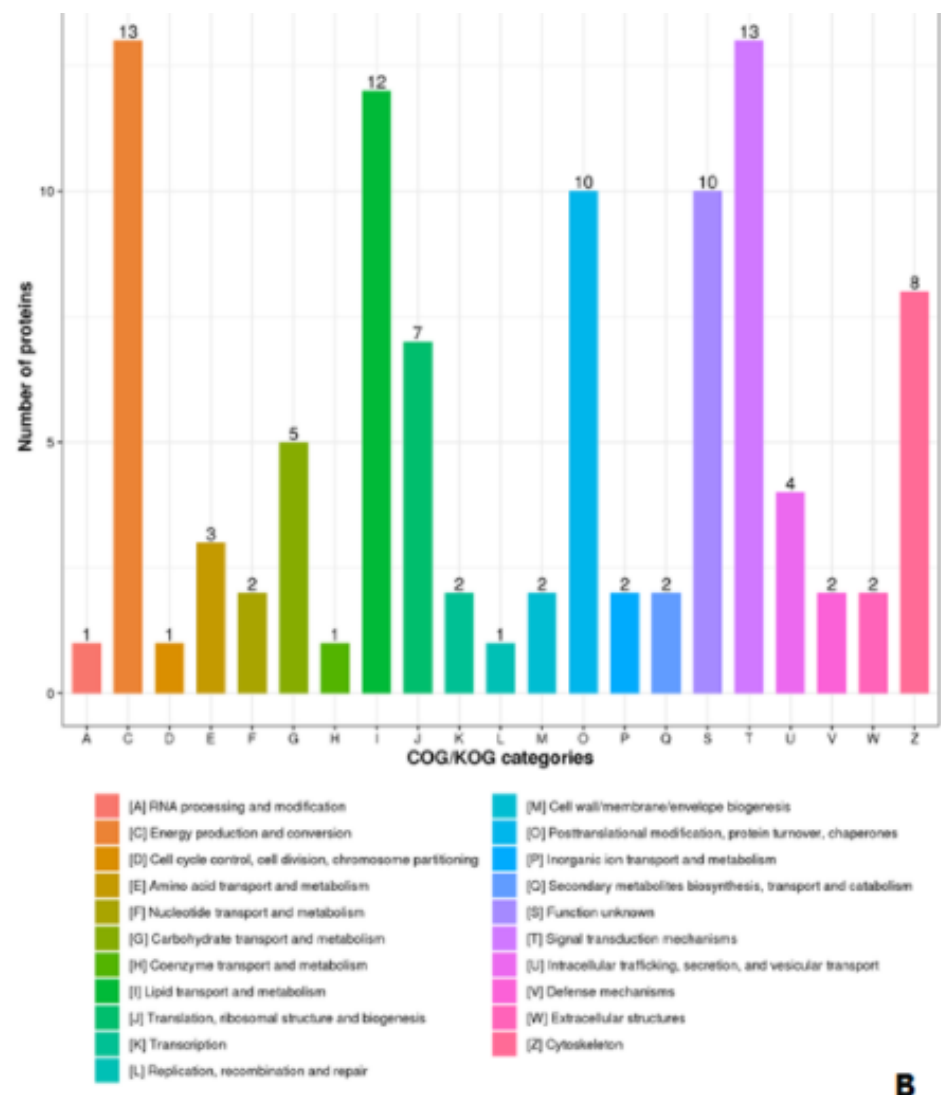

Figure 2

A: Distribution chart of the subcellular localization of the differentially expressed proteins. B: Distribution chart of $\mathrm{COG} / \mathrm{KOG}$ functional classification of the differentially expressed proteins. 


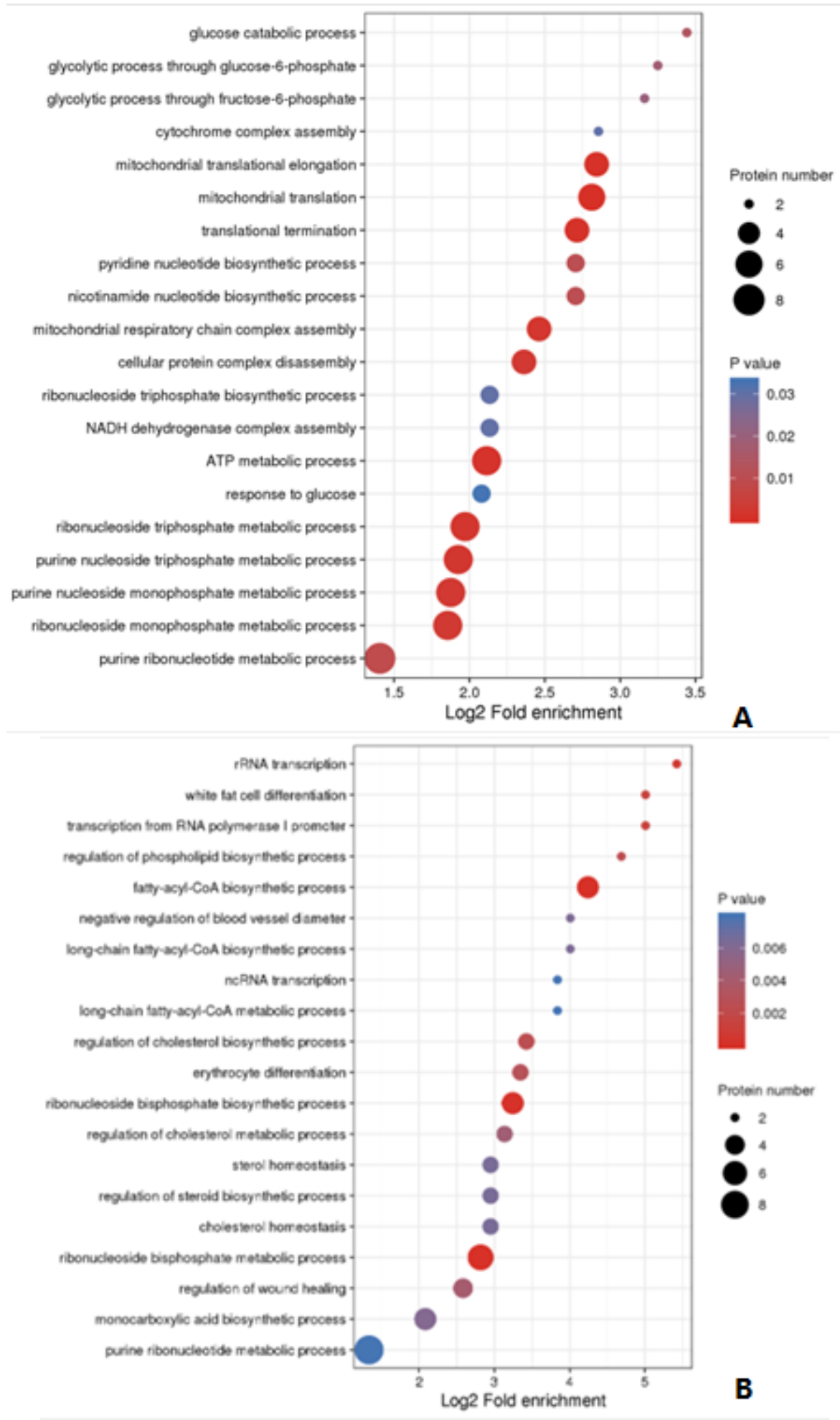

\section{Figure 3}

Enrichment distribution bubble charts of differentially expressed proteins regarding biological processes in the GO functional classification of the experimental group vs. the positive control group (A pertains to upregulation and $B$ pertains to downregulation). The circles in the figure represent the $G O$ classification in which the differentially expressed proteins were located. The circle size represents the amount of protein, and enrichment increases along the $\mathrm{X}$-axis. 


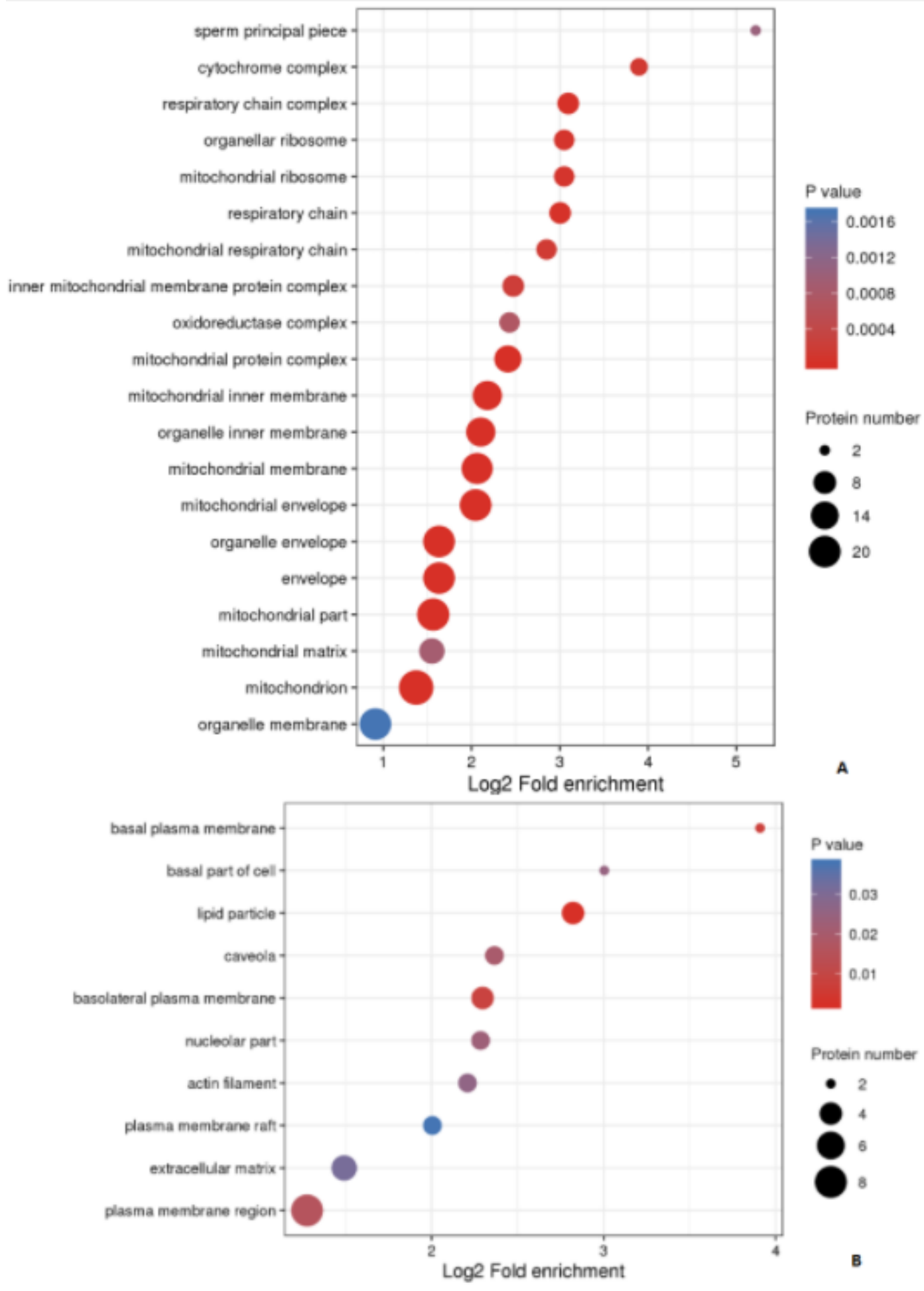

\section{Figure 4}

Enrichment distribution bubble charts of differentially expressed proteins of cell components in the GO functional classification of the experimental group vs. the positive control group (A pertains to upregulation and $B$ pertains to downregulation). The circles in the figure represent the $\mathrm{GO}$ classification in which the differentially expressed proteins are located. The circle size represents the amount of protein, and the enrichment increases along the $\mathrm{X}$-axis. 

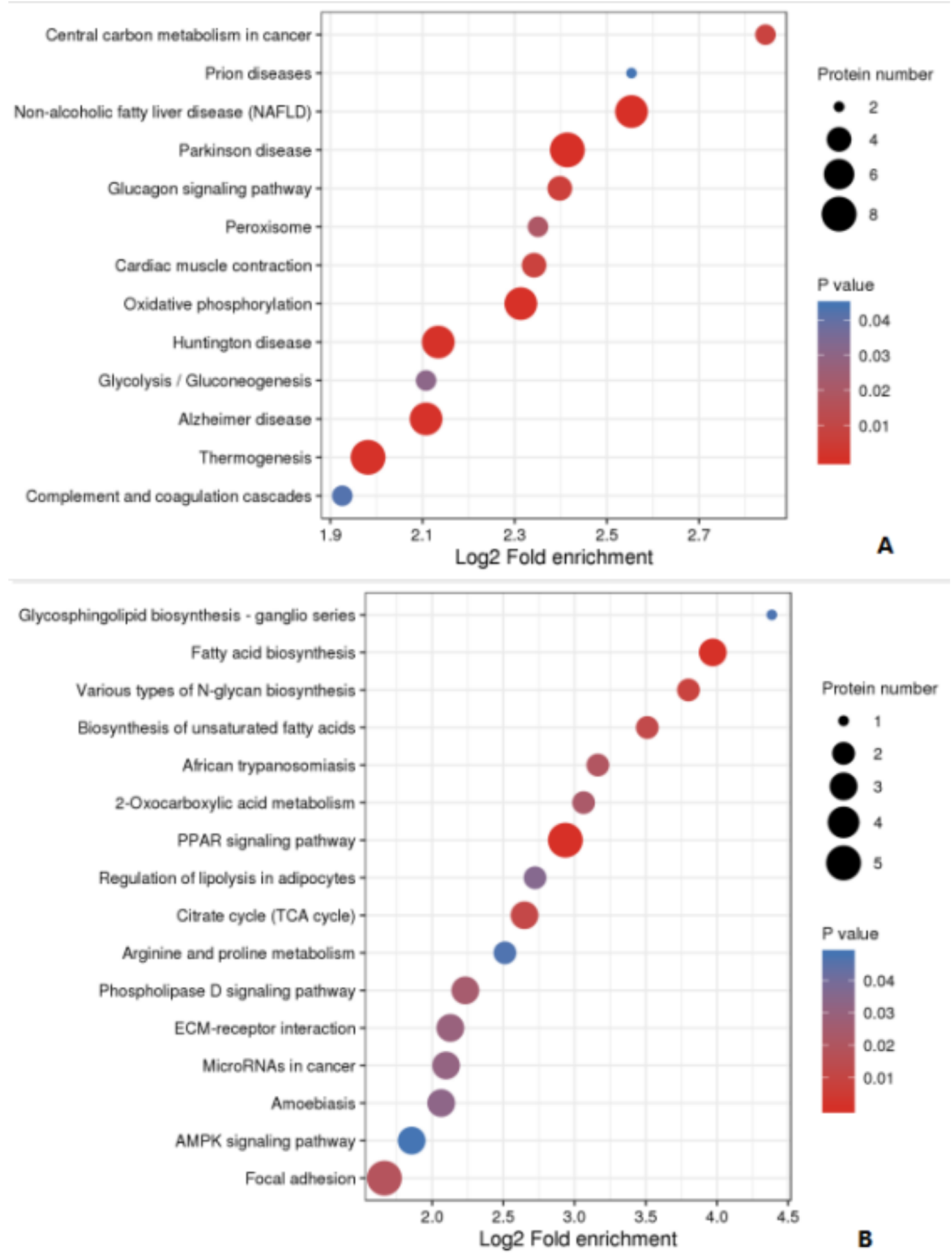

\section{Figure 5}

Enrichment distribution bubble charts of differentially expressed proteins regarding KEGG pathways in the GO functional classification of the experimental group vs. the positive control group (A pertains to upregulation and $\mathrm{B}$ pertains to downregulation). The circles in the figure represent the $\mathrm{GO}$ classification in which the differentially expressed proteins are located. The circle size represents the amount of protein, and the enrichment increases along the $\mathrm{X}$-axis. 


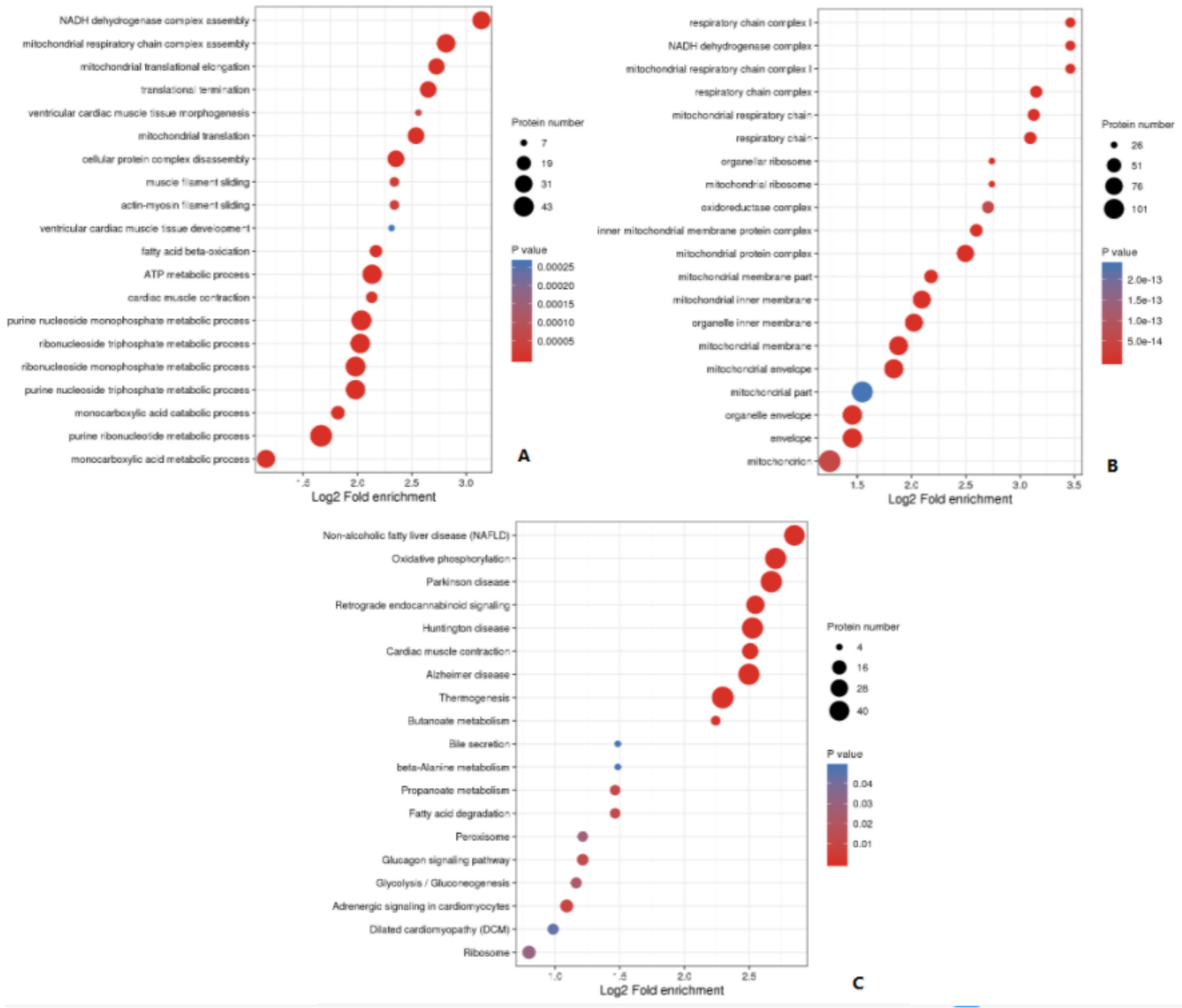

Figure 6

Enrichment distribution bubble charts of downregulated differentially expressed proteins in the GO functional classification of the positive control group vs. the negative control group (A pertains to Biological processes, $B$ pertains to cell components, and $C$ pertains to KEGG pathways). The circles in the figure represent the $\mathrm{GO}$ classification in which the differentially expressed proteins were located. The circle size represents the amount of protein, and the enrichment increases along the $\mathrm{X}$-axis.

\section{Supplementary Files}

This is a list of supplementary files associated with this preprint. Click to download.

- Table1.png 